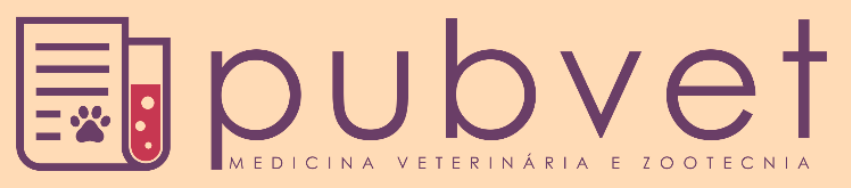

https://doi.org/10.31533/pubvet.v12n12a232.1-4

\title{
Uso de dipirona em gatos na América do Sul: Pesquisa
}

\section{Luciana Gonçalves Teixeira ${ }^{1^{*}}$, Luiza Tonietto Mangini' ${ }^{2}$, Pery Soares Baccin ${ }^{2}$,

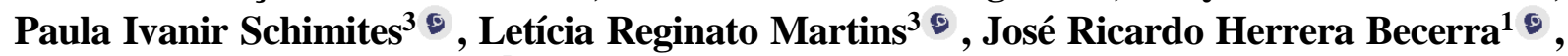 André Vasconcelos Soares ${ }^{4}$}

${ }^{1}$ Mestre, Doutorando(a) em Ciências Veterinárias, UFRGS - Universidade Federal do Rio Grande do Sul.

${ }^{2}$ Aluno de graduação, UFSM - Universidade Federal de Santa Maria

${ }^{3}$ Mestre, Doutoranda em Cirurgia e Clínica Veterinárias, UFSM - Universidade Federal de Santa Maria

${ }^{4}$ Doutor, Professor adjunto do Departamento de Clínica de Pequenos Animais, UFSM - Universidade Federal de Santa Maria

*Autor para correspondência, E-mail: lucianateixeira@gmail.com

\begin{abstract}
RESUMO. O objetivo deste trabalho foi relatar o uso da dipirona como analgésico em felinos domésticos por médicos veterinários sul americanos, referente à: uso, dose, frequência, período de utilização e efeitos colaterais observados. Foi realizado um questionário virtual através da plataforma Google que foi enviado para profissionais veterinários. O mesmo foi respondido por um total de 288 médicos veterinários. Destes, $81,9 \%$ afirmaram utilizar o fármaco na rotina clínica. A dose de $25 \mathrm{mg} / \mathrm{kg}^{-1}$ foi a mais citada, sendo que $32,3 \%$ administram-na duas vezes ao dia (BID), 19,1\% uma vez ao dia (SID) e $10,1 \%$ três vezes ao dia (TID). Outra dose frequentemente utilizada é $12,5 \mathrm{mg} / \mathrm{kg}$ 1, BID (15,6\%), SID $(6,3 \%)$ e TID (4,9\%). Ainda, $11,8 \%$ dos entrevistados utilizam a medicação em doses e frequências variadas. O período de administração mais utilizado é de 1 a 3 dias $(64,6 \%)$. Os efeitos adversos mais observados e relacionados ao uso da dipirona por $27,2 \%$ dos entrevistados são sialorreia, vômito, agitação, reação alérgica, apatia, parada cardíaca, hipotensão entre outros.
\end{abstract}

Palavras chave: analgesia, dipirona, gatos, questionário

\section{Use of dipyrone in cats in South America: Research}

\begin{abstract}
The aim of this work was to report the use of dipyrone as an analgesic in domestic cats by South American veterinarians regarding dose, posology, period of use and the observed side effects. A virtual survey was conducted through the Google platform and was sent to veterinary professionals. The questionnaire was answered by a total of 288 veterinarians. Of these, $81.9 \%$ reported using the drug in their clinical routine. The 25 $\mathrm{mg} / \mathrm{kg}^{-1}$ dose was the most cited, with $32.3 \%$ of the respondents affirming to administer it twice a day (BID), $19.1 \%$ once a day (SID) and $10.1 \%$ three times a day (TID). Another dose oftenly used is $12.5 \mathrm{mg} / \mathrm{kg}^{-1}$, BID (15.6\%), SID (6.3\%) and TID (4.9\%). Still, $11.8 \%$ of the interviewees use the drug in varied doses and frequencies. The most commonly used administration period is 1 to 3 days $(64.6 \%)$. The most observed adverse effects related to the use of dipyrone by $27.2 \%$ of the interviewees are sialorrhea, vomiting, agitation, allergic reaction, apathy, cardiac arrest, hypotension, among others.
\end{abstract}

Keywords: analgesia, cats, dipyrone, survey

\section{Uso de dipirona en gatos en América del Sur: Investigación}

RESUMEN. El objetivo de este trabajo fue evaluar el uso de dipirona en la rutina clínica de los médicos veterinarios de américa latina, en lo que se refiere a: uso, dosis, frecuencia, 
período de utilización y efectos colaterales observados. Se realizó un cuestionario a través de la plataforma Google que fue enviado a profesionales veterinarios. En total 288 médicos veterinarios sudamericanos respondieron sobre el uso de dipirona como analgésico en felinos domésticos. El 81,9\% (236) de ellos respondieron que utilizan el fármaco en la rutina clínica. De los entrevistados que usan dipirona, la dosis de $25 \mathrm{mg} / \mathrm{kg}^{-1}$ fue la más citada, donde el 32,3\% administra dos veces al día (BID), el 19,1\% administra una vez al día (SID) y el 10,1\% prescriben tres veces al día (TID). Otra dosis frecuentemente utilizada es $12,5 \mathrm{mg} / \mathrm{kg}^{-1}$, BID (15,6\%), SID (6,3\%) y TID (4,9\%). Además, el 11,8\% de los entrevistados manifestaron utilizar la medición en dosis y frecuencias variadas. En cuanto a la duración del tratamiento con dipirona, el 64,6\% prescribe entre 1 a 3 días, 31,3\% de 3 a 5 días y el $4,2 \%$ utilizan por un tiempo superior a 5 días. En cuanto a los posibles efectos adversos, el 27,2\% observaron efectos colaterales a los cuales se relacionan al uso del fármaco, siendo ellos: sialorrea, vómito, agitación, reacción alérgica, apatía, paro cardíaco, hipotensión, entre otros.

Palabras clave: analgesia, dipirona, gatos, cuestionario

\section{Introdução}

Os anti-inflamatórios não esteroides (AINEs) são uma das principais classes de drogas analgésicas utilizadas em medicina veterinária. A dipirona, ou metamizole, pertence a esta classe e é considerada inibidora da cicloxigenase-3. O metamizole tem seu metabolismo no fígado e neste local é fracionado em numerosos produtos metabólicos sendo o principal 4metilaminoantipirina (MAA), que é então metabolizado a 4-formilaminoantipirina (FAA; um metabolito final) e 4-aminoantipirina (AA). O AA é, ainda, metabolizado a 4-acetilaminoantipirina (AAA) (Giorgi et al. 2018). Devido ao seu caráter quieto e expressão sutil de dor, os gatos recebem relativamente menos analgésicos em comparação com outros animais (Giorgi et al. 2018).

Em cães, o metamizole possui propriedades analgésicas, antipiréticas e espasmolíticas sem apresentar as limitações usualmente observadas com o uso de opioides ou outros AINEs. Quando parte de um sistema analgésico multimodal, o metamizol pode reduzir consumo de opioides e, consequentemente, seus efeitos (Torres et al. 2001). Acredita-se que a dipirona, necessite de conjugação com glicuronídeos previamente à excreção renal e, por isso, sugere-se cautela ou até mesmo a não utilização desta medicação em gatos, uma vez que faltam estudos acerca da toxicidade e efeito analgésico da droga nesta espécie. Portanto, seu uso em felinos é mais empírico, promovendo dúvidas nos médicos veterinários quanto à segurança e à eficácia de seu uso nesta espécie.

O objetivo do trabalho foi fazer um levantamento do uso de dipirona como analgésico em felinos, as doses e frequência de utilização, além dos efeitos colaterais observados por médicos veterinários brasileiros.

\section{Material e Métodos}

Para a realização deste levantamento, um questionário virtual, realizado através da plataforma Google, contendo quatro perguntas foi confeccionado. Foi averiguado se os médicos veterinários entrevistados (a) utilizavam a dipirona para o tratamento da dor de gatos; (b) a dose e frequência de administração utilizada; (c) o período de utilização normalmente prescrito, e (d) se algum efeito adverso já havia sido observado com o uso da dipirona em gatos, e qual. Todas as questões eram de múltipla escolha.

A questão b incluía 7 opções, considerando as doses de 12,5 e $25 \mathrm{mg} / \mathrm{kg}^{-1}$, ambas podendo ser administradas em intervalos de 24 (SID), 12 (BID) ou 8 horas (TID), além de uma opção "outro", que poderia ser indicada em um campo acessório à questão b. Na questão c, os entrevistados poderiam optar pelo uso por 3 dias, de 3 a 5 dias ou maior do que 5 dias, nestas mesmas doses.

O questionário foi aplicado num período de 30 dias a partir do compartilhamento entre médicos veterinários de diferentes instituições do Brasil e países da América do Sul.

\section{Resultados}

Ao todo, 288 médicos veterinários responderam o questionário, 219 brasileiros e 69 sul-americanos. Destes, 81,9\% (236) afirmaram utilizar a dipirona como analgésico em sua rotina clínica, enquanto $18,1 \%$ (52) disseram não fazer uso do fármaco. A maior parte dos entrevistados (77\%; 222) administra dipirona na dose de 25 $\mathrm{mg} / \mathrm{kg}^{-1}$, variando, contudo, a posologia. Enquanto 
41\% (93/222) administram-na a cada 12 horas (BID), 24\% (55/222) utilizam-na a cada 24 horas (SID) e, somente $13 \%$ (29/222) a cada 8 horas (TID). A segunda dose informada mais utilizada foi de $12,5 \mathrm{mg} / \mathrm{kg}^{-1}$, usada por $11,2 \%$ (32) dos respondentes, os quais administram-na SID (56\%; 18/32) ou BID $(43 \% ; 14 / 32)$. Outras doses e frequências também foram informadas por $7 \%$ (21/288) dos entrevistados. Dentre essas, destacase a de $20 \mathrm{mg} / \mathrm{kg}^{-1}$ e $28 \mathrm{mg} / \mathrm{kg}^{-1}(1,7$ e $1,0 \%$, respectivamente), $16 \mathrm{mg} / \mathrm{kg}^{-1}(0,7 \%)$ e as doses de $10,14,15$, e $22 \mathrm{mg} / \mathrm{kg}^{-1}$, informadas por 1 a 2 pessoas, cada.

O tempo total de tratamento com dipirona varia entre 1 a 3 dias $(64,6 \% ; 186)$, para a maioria dos médicos veterinários, 3 a 5 dias $(31,3 \%$; 90) e um período superior a cinco dias, realizado pela minoria dos entrevistados $(4,2 \% ; 12)$.

$\mathrm{Na}$ maioria das vezes em que utilizaram dipirona, os entrevistados apontam não ter evidenciado nenhum efeito adverso $(72,8 \%$; 209). Contudo, 27,2\% dos respondentes relacionaram algum efeito indesejável com o uso da droga, sendo a sialorreia, o efeito mais observado (59\%), acompanhada ou não de vômito (6\%), agitação $(3,6 \%)$ ou apatia $(2,4 \%)$. Náusea e vômito foram observados em 9,6 e 7,2\% dos casos, respectivamente. Outros efeitos como hemólise intravascular e reação alérgica foram relacionados à dipirona por $6 \%$ e $3,6 \%$, respectivamente. Ainda, menos de $0,02 \%$ (8/236) dos entrevistados relataram parada cardiorrespiratória, hipotensão, alteração de enzimas hepáticas, diarreia e leucopenia associados à dipirona, distribuídos entre esses efeitos.

\section{Discussão}

Os resultados dessa pesquisa revelam que a dipirona é amplamente utilizada como analgésico em gatos. Todavia, ainda há grande variação de doses e posologia informada. Alguns autores recomendam a utilização de 12 a $25 \mathrm{mg} / \mathrm{kg}^{-1}$ BID aplicados por um máximo de 2 dias e com cautela (Gaynor \& Muir 2014; Mathews et al. 2014). Isso se deve ao fato de a dipirona ser um composto fenólico e de os gatos apresentarem limitações à metabolização dessas substâncias, como apenas duas isoformas da enzima uridina difosfato glucuronosiltransferase, principal catalisadora da glicuronidação (Court 2013), e 8 sulfidrilas em seus eritrócitos, o que torna suas células mais instáveis e suscetíveis à oxidação (Harvey \& Bruss 2008). Assim, um aumento no tempo de excreção e uma potencial exposição dos eritrócitos desses animais à ação de metabólitos capazes de induzir a lesões oxidativas nessas células pode ser esperado.

Giorgi et al. (2018) demonstraram que gatos convertem dipirona na dose de $25 \mathrm{mg} / \mathrm{kg}^{-1}$ em seus metabólitos ativos, tal como os humanos, e que o MAA, metabólito relacionado à analgesia, apresenta uma meia-vida de aproximadamente 6 horas, independentemente da via de administração. Esse dado é importante, pois indica que a administração de $25 \mathrm{mg} / \mathrm{kg}^{-1}$ BID de dipirona, realizada pela maior parte dos médicos veterinários entrevistados, está adequada. Contudo, as demais doses e posologias informadas, exceto por $25 \mathrm{mg} / \mathrm{kg}^{-1} \mathrm{TID}$, parecem ser ineficazes. Corroborando com isto, têm-se que trabalhos em cães apontam que a mínima dose capaz de promover analgesia é $25 \mathrm{mg} / \mathrm{kg}^{-1}$ (Zanuzzo et al. 2015).

Os estudos sobre analgesia em cães evidenciam que o tratamento com dipirona pode ser uma opção para a dor, inclusive de origem póscirúrgica de intensidade leve a moderada, mas que seu efeito tende a ser mais evidenciado quando combinada com outras drogas analgésicas (Teixeira et al. 2013; Zanuzzo et al. 2015). Entretanto, não há estudos demonstrando a eficácia analgésica da dipirona em gatos. Apesar disso, a julgar pelo efeito já descrito em humanos e cães e pela farmacocinética deste fármaco na espécie felina, além da experiência prática veterinária, o uso da dipirona deve estar relacionado à promoção de analgesia também nessa espécie, restando saber as doses adequadas para este efeito. Os guias reconhecimento e tratamento da dor recomendam o uso da dipirona para a analgesia em gatos (Mathews et al. 2014).

\section{Conclusão}

Conclui-se que o uso da dipirona é amplamente difundido entre o público médico veterinário avaliado, e sua forma de administração em gatos ainda é dependente da conduta de cada profissional, necessitando de maiores estudos teóricos que definam consenso para tal, sendo a sua administração preterida quando comparada ao uso de AINES nesta espécie.

\section{Referências bibliográficas}

Court M.H. 2013. Feline drug metabolism and disposition: pharmacokinetic evidence for species differences and molecular 
mechanisms. The Veterinary Clinics of North America. Small Animal Practice 43, 10391054.

Gaynor J.S. \& Muir W.W. 2014. Appendix. In: Handbook of Veterinary Pain Management (eds. by J.S. G \& W.W. M). Elsevier, St Louis, USA.

Giorgi M., Łebkowska-Wieruszewska B., Lisowski A., Owen H., Poapolathep A., Kim T.W. \& Vito V. 2018. Pharmacokinetic profiles of the active metamizole metabolites after four different routes of administration in healthy dogs. Journal of veterinary pharmacology and therapeutics 41, 428-436.

Harvey J.W. \& Bruss M.L. 2008. The erythrocyte. In: Clinical Biochemistry of Domestic Animas (eds. by Kaneko J, Harvey J \& Bruss M). Elsevier., USA.

Mathews K., Kronen P.W., Lascelles D., Nolan A., Robertson S., Steagall P.V.M., Wright B. \& Yamashita K. 2014. Guidelines for recognition, assessment and treatment of pain: WSAVA global pain council members and coauthors of this document. Journal of Small Animal Practice 55, E10-E68.

Teixeira R.C.R., Monteiro E.R., Campagnol D., Coelho K., Bressan T.F. \& Monteiro B.S. 2013. Effects of tramadol alone, in combination with meloxicam or dipyrone, on postoperative pain and the analgesic requirement in dogs undergoing unilateral mastectomy with or without ovariohysterectomy. Veterinary Anaesthesia and Analgesia 40, 641-649.

Torres L.M., Rodríguez M.J., Montero A., Herrera J., Calderón E., Cabrera J., Porres R., Rosalia M., Martínez T. \& Gómez J.L. 2001. Efficacy and safety of dipyrone versus tramadol in the management of pain after hysterectomy: a randomized, double-blind, multicenter study. Regional Anesthesia and Pain Medicine 26, 118-124.

Zanuzzo F.S., Teixeira-Neto F.J., Teixeira L.R., Diniz M.S., Souza V.L., Thomazini C.M. \& Steagall P.V.M. 2015. Analgesic and antihyperalgesic effects of dipyrone, meloxicam or a dipyrone-meloxicam combination in bitches undergoing ovariohysterectomy. The Veterinary Journal 205, 33-37.

Recebido: 6 novembro, 2018 .

Aprovado: 27 novembro, 2018.

Publicado: 27 dezembro, 2018.

Licenciamento: Este artigo é publicado na modalidade Acesso Aberto sob a licença Creative Commons Atribuição 4.0 (CC-BY 4.0), a qual permite uso irrestrito, distribuição, reprodução em qualquer meio, desde que o autor e a fonte sejam devidamente creditados. 